\title{
Seasonal and Radial Trends in Saturn's Thermal Plasma Between the Main Rings
} and Enceladus

\author{
M.K. Elrod ${ }^{1,2}$, W-L Tseng ${ }^{3}$, A. K. Woodson ${ }^{1}$, R. E. Johnson ${ }^{1}$ \\ ${ }^{1}$ University of Virginia, Charlottesville, VA \\ ${ }^{2}$ National Institute of Aerospace, Hampton, VA \\ ${ }^{3}$ Southwest Research Institute, San Antonio, TX
}

\section{Abstract}

16 of Saturn's magnetosphere, moons, and rings. Recently we showed that the

17 magnetospheric plasma between the main rings and Enceladus exhibited a time

18 dependence that we attributed to a seasonally variable source of oxygen from the main

19 rings (Elrod et al., 2012). Such a temporal variation was subsequently seen in the

20 energetic ion composition (Christon et al., 2013). Here we include the most recent

21 measurements by the Cassini Plasma Spectrometer (CAPS) in our analysis (Elrod et al.,

22 2012) and modeling (Tseng et al., 2013a) of the temporal and radial dependence of the

23 thermal plasma in the region between the main rings and the orbit of Enceladus. Data

24 taken in 2012, well past equinox for which the northern side of the main rings were

25 illuminated, appear consistent with a seasonal variation. Although the thermal plasma in

26 this region comes from two sources, the extended ring atmosphere and the Enceladus

27 torus that have very different radial and temporal trends, the heavy ion density is found to

28 exhibit a steep radial dependence that is similar for all years examined. Using our

29 chemical model, we show that this dependence requires a radial dependence for

30 Enceladus torus than differs from recent models or, more likely, enhanced heavy ion

31 quenching with decreasing distance from the edge of the main rings. We examine the

32 possible physical processes and suggest that the precipitation of the inward diffusing

33 high-energy background radiation onto the edge of the main rings could play an

34 important role. 


\section{1. Introduction}

36 The thermal plasma in the inner magnetosphere, from just outside the main rings

37 to just inside the orbit of Enceladus $\left(\sim 2.4 R_{S}-3.8 R_{S} ; 1 R_{S}=1\right.$ Saturn Radius $=$

38 60,300km), is studied using Cassini Plasma Spectrometer (CAPS) data from 2004

39 through 2012. The goal of this paper is to further examine the temporal and radial

40 variation in the heavy ion plasma (Elrod et al., 2012). The $\mathrm{O}_{2}^{+}$observed by the CAPS

41 instrument over the main rings (Tokar et al., 2006) was suggested to be formed from $\mathrm{O}_{2}$

42 produced by photo-decomposition of the icy ring particles (Johnson et al., 2006a). A

43 fraction of this $\mathrm{O}_{2}$ is subsequently scattered, forming an extended ring atmosphere, in

44 which the molecules are ionized contributing to the plasma in Saturn's inner

45 magnetosphere (e.g., Johnson et al., 2006a; Martens et al., 2008). Since such a source

46 depends on the illumination of the ring plane, the plasma density was predicted to vary

47 over Saturn's orbit as the ring plane illumination varied from the southern hemisphere

48 through equinox to the northern hemisphere (Tseng et al., 2010; 2013a). Water group

49 ions, labeled here as $\mathrm{W}^{+}\left(\mathrm{O}^{+}, \mathrm{OH}^{+}, \mathrm{H}_{2} \mathrm{O}^{+}\right.$, and $\left.\mathrm{H}_{3} \mathrm{O}^{+}\right)$are also directly formed in this

50 region by ionization of neutrals in the Enceladus torus (Cassidy et al, 2010; Smith et al.

51 2010; Tseng et al., 2012). While this source probably does not exhibit a seasonal

52 variation, there is evidence that it fluctuates by up to a factor of four (Smith et al., 2010)

53 and appears to depend on the position of Enceladus in its orbit (Hedman et al. 2013).

54 In Elrod et al. (2012) we used CAPS data from SOI to 2010 to confirm that the

55 main rings are an important source of $\mathrm{O}_{2}{ }^{+}$and $\mathrm{O}^{+}$ions inside the orbit of Mimas. We also

56 showed that there was a steep drop in $\mathrm{O}_{2}{ }^{+}$density, as well as in the total heavy ion

57 thermal plasma, over that time period. Consistent with this, the Magnetospheric 
58 Imagining Instrument (MIMI) recently reported a decrease from SOI through 2010 in

59 energetic $\mathrm{O}_{2}^{+}$density as compared to the $\mathrm{W}^{+}$density, followed by a recovery in 2011-

602012 (Christon et al., 2013). More recently a seasonal variation was reported equatorial

61 electron densities measured by the Radio and Plasma Wave Instrument (RPWS) (Persoon

62 et al. 2013a).

63 The present study of the thermal plasma extends our earlier time frame by

64 including the 2012 data in order to determine whether or not the density increases after

65 equinox. Because seasonal variations were assumed to be due to a ring atmosphere source

66 superimposed on the Enceladus torus source, the seasonal variation might be expected to

67 decrease with distance from the main rings consistent with models of the ring

68 atmosphere. Surprising for all the years studied, the total heavy ion density, as well as

69 that for the individual $\mathrm{O}_{2}^{+}$and $\mathrm{W}^{+}$ions, increases relatively steeply with increasing

70 distance from Saturn. This is the case even though the proposed seasonal variation is

71 thought to be primarily due to molecules scattered from the ring atmosphere. After

72 presenting the new results, we consider the implications of these findings.

73

\section{2. Analysis}

75 In order to study the temporal and radial variation in the density and composition

76 of the thermal plasma, we examined passes from SOI in 2004, two in 2007 (doy 162 \&

77 178/179), three in 2010 (doy 062, 154, and 170), and two in 2012 (doy 087 and 105) in

78 the region between $\sim 2.4 R_{S}-3.8 R_{S}\left(1 R_{S}=1\right.$ Saturn Radius $\left.=60,300 \mathrm{~km}\right)$. All of these

79 passes had a periapsis within the Enceladus orbit at $\sim 4.0 R_{S}$ and had near equatorial

80 segments which we focus on here. In Fig. 1 we show both the orbital paths in the $R$ vs. $Z$ 
81 plane and the local time for each of the passes used in this study. Although there is a

82 significant spread in local time, with the exception of SOI, all the passes were on the

83 dayside, reducing the importance of the suggested night to day variations seen by the

84 Cassini Radio and Plasma Wave Science (RPWS) Langmuir probe (Holmberg et al.,

85 2013).

86

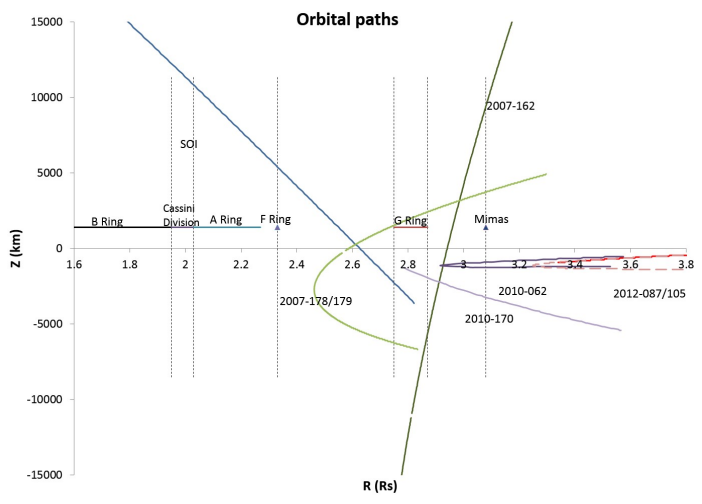

(a)

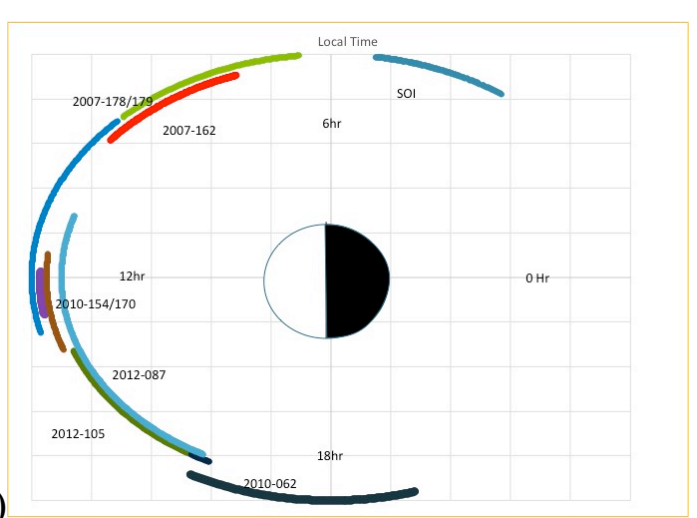

87 (b)

88 Figure 1. Orbits used in this study: a) Position in equatorial radius, $R$ in $R_{s}(1 \mathrm{Rs} \approx 60,300 \mathrm{~km}$ in the

89 equatorial plane) vs. distance above the equator, $Z$ in $\mathrm{km}$; for consistency in the comparisons and to

90 maximize the count rate, the data presented below comes from the near equatorial segments $(Z$ within

$91 \pm 5,000 \mathrm{~km})$. b) Local times during each orbit. With the exception of SOI, most of the data was taken on the 92 dayside between dawn and dusk.

95 data, the correction for the significant background in this region, and the fitting of the

96 measured energy spectrum to obtain the total ion densities as well as the $\mathrm{O}_{2}^{+}$and $\mathrm{W}^{+}$

97 fractions. These details are not repeated here. Unlike in Elrod et al. (2012) we only

98 analyzed CAPS singles data taken close to the equator, having a minimum number of A-

99 cycles, and at least 400 counts above background in order to reduce the scatter in the

100 data. We also included the 2012 data, which was not available earlier. Although there are

101 variations in the total heavy ion density observed between those passes in Fig. 1 
102 occurring in the same year, which we will examine in the future, these variation are

103 typically smaller than the variations between years. Therefore, we show in Fig. 2 the

104 average of the total heavy ion density for each year vs. radial distance from Saturn for

105 those segments close to the equator $(Z<5000 \mathrm{~km})$.

106

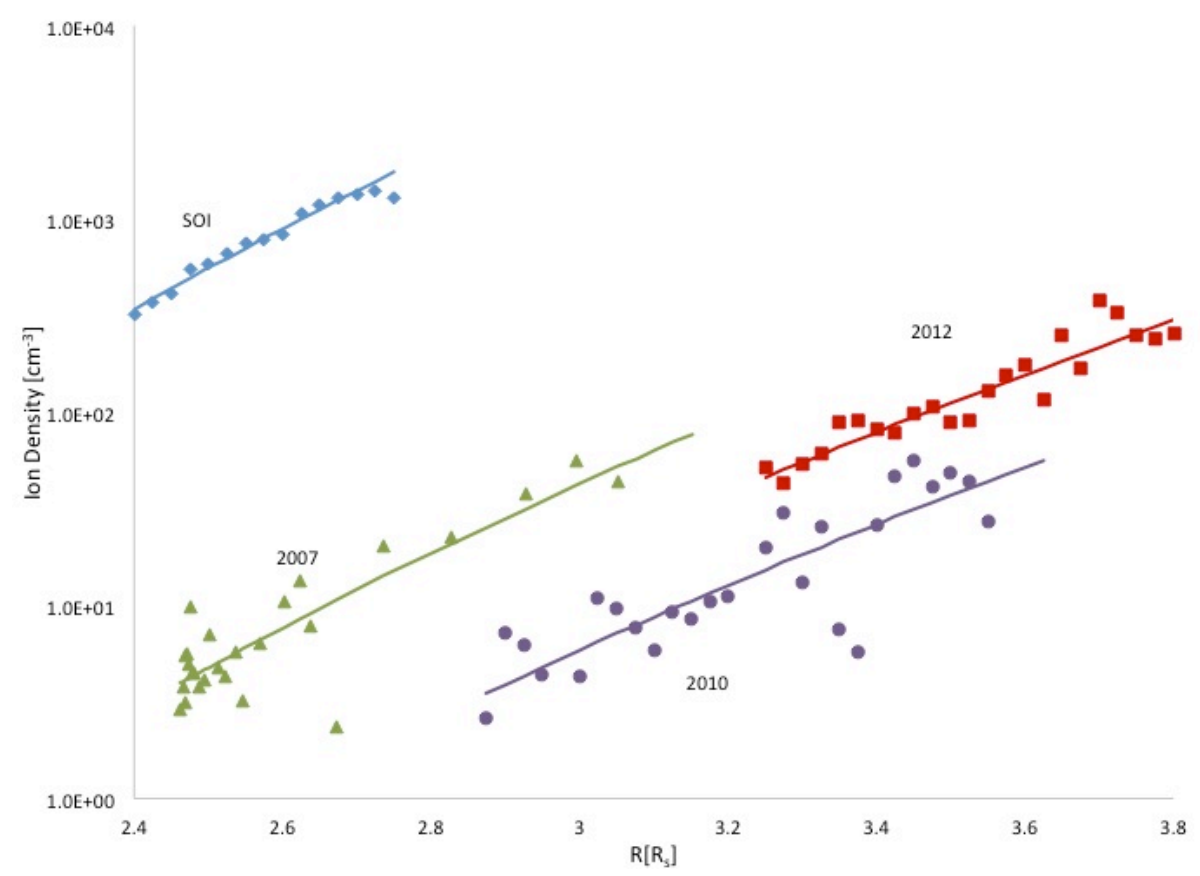

108 Figure 2. Total heavy ion densities vs. $R$ in $R_{s}$ from 2004 through 2012 averaged over orbits for each year

109 in Fig. 1a: SOI in 2004 (blue diamonds), 2007 (green triangles), 2010 (purple dots), and 2012 (red squares).

110 Lines are fits: $n_{i}=c\left(R / 3 R_{S}\right)^{12}$; where $\mathrm{c}$ is the extrapolated value $n_{i}\left(3 R_{S}\right)$ : (SOI) blue $\left[c=4.94 \times 10^{3} / \mathrm{cm}^{3}\right], 2007$

111 green $\left[c=42.5 / \mathrm{cm}^{3}\right], 2010$ purple $\left[c=5.85 / \mathrm{cm}^{3}\right]$, and 2012 orange $\left[c=17.5 / \mathrm{cm}^{3}\right]$.

112

113

It is seen in Fig. 2 that there is a clear decrease in the heavy ion density from 2004

114 through 2010 and an increase from 2010 to 2012. Therefore, the lowest densities occur in

1152010 , the orbit closest to equinox (11 August, 2009) with apparent recovery through

116 2012. It is also seen that all densities, regardless of year, increase radially outward. While

117 there are significant changes in the magnitude of the densities between the years, the

118 radial dependence of each of these sets is remarkably similar. In order to better quantify

119 the data we fit a power law in equatorial distance from Saturn, $R$, to each of these 
120 averaged data sets. The steep variation is roughly fit using the form $n_{i}=c\left(R / 3 R_{S}\right)^{12}$.

121 Because the radial range is narrow, powers varying from $\sim 10-14$ also give reasonable

122 descriptions. We use a power of 12 in the following and note that the measured radial

123 dependence is relatively steep.

124 As discussed below, the SOI data is dominated by $\mathrm{O}_{2}{ }^{+}$, likely from the extended

125 ring atmosphere, with temperatures close to the fresh ion pick-up temperature, whereas

126 the later data sets, closer to equinox, are dominated by $\mathrm{W}^{+}$, likely from the Enceladus

127 torus, but with low ion temperatures (Elrod et al., 2012). In spite of the different

128 compositions and temperatures, there is a striking similarity in the radial dependence in

129 all of the years studied.
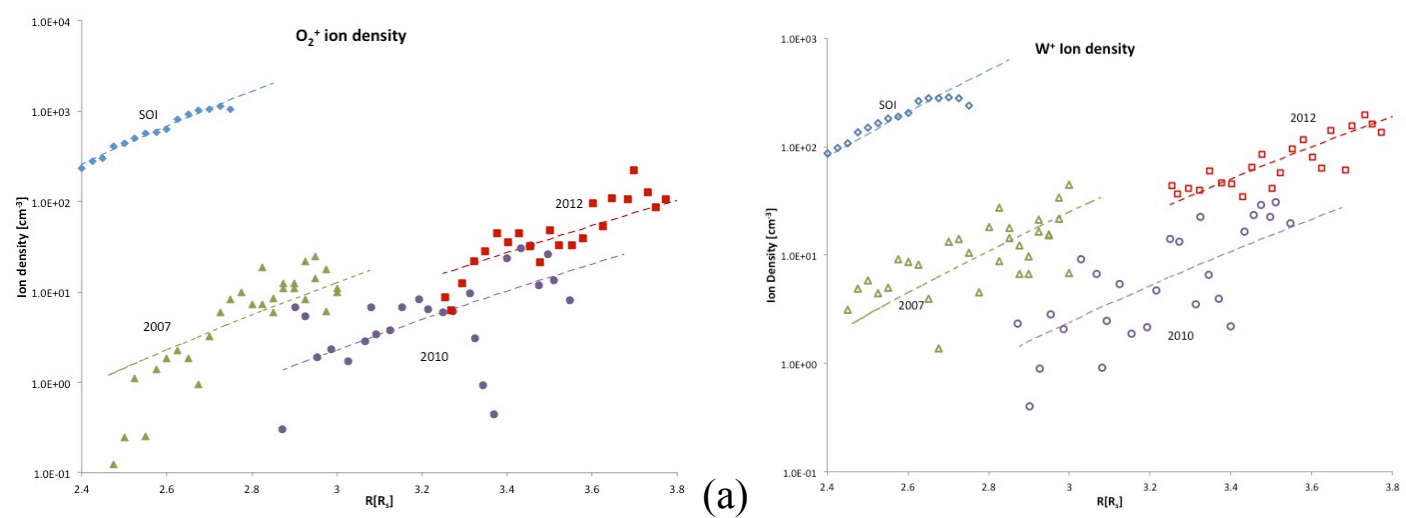

Figure 3. Averaged densities for each year: (a) $\mathrm{O}_{2}^{+}$, closed symbols; (b) $\mathrm{W}^{+}\left(\mathrm{O}^{+}, \mathrm{OH}^{+}, \mathrm{H}_{2} \mathrm{O}^{+}, \mathrm{H}_{3} \mathrm{O}^{+}\right)$, open symbols: SOI in 2004 (blue diamonds), 2007 (green triangles), 2010 (purple dots), 2012 (red squares). As in Fig. 2 the lines are fits: $n_{i}(R)=c\left(R / 3 R_{S}\right)^{12}$. The extrapolated values $\mathrm{c} \approx n_{i}\left(3 R_{S}\right)$ in $\mathrm{cm}^{-3}$ for $\mathrm{O}_{2}^{+}$and $\mathrm{W}^{+}$ respectively: $\left(2004: 3.8 \times 10^{3}, 1.2 \times 10^{3}\right),(2007: 12.8,25.0),(20010 ; 2.3,2.4)$ and $(2012: 6.06,11.2)$.

Using the analysis in Elrod et al. (2012) with the selectivity discussed here, we

137 also show the yearly averaged $\mathrm{O}_{2}^{+}$and $\mathrm{W}^{+}$components of the density in Figs. $3 \mathrm{a}$ and $\mathrm{b}$.

138 Because the peaks in the CAPS singles spectra overlap significantly, these data have a

139 much larger scatter, but still exhibit a radial dependence roughly similar to that in Fig. 2.

140 Therefore, we also fit each set using $n_{i}=c\left(R / 3 R_{S}\right)^{12}$. It is seen to capture the general 
141 trend with the exception of the $\mathrm{O}_{2}{ }^{+} 2007$ data at the smallest values of $R$. None of these

142 fits are unique, as the $\mathrm{O}_{2}^{+}$data can be better fit on average with a slightly smaller power

143 and the $\mathrm{W}^{+}$with a slightly larger power. Here we use the rough fits in Figs. 2 and 3 to

144 guide the discussion below.

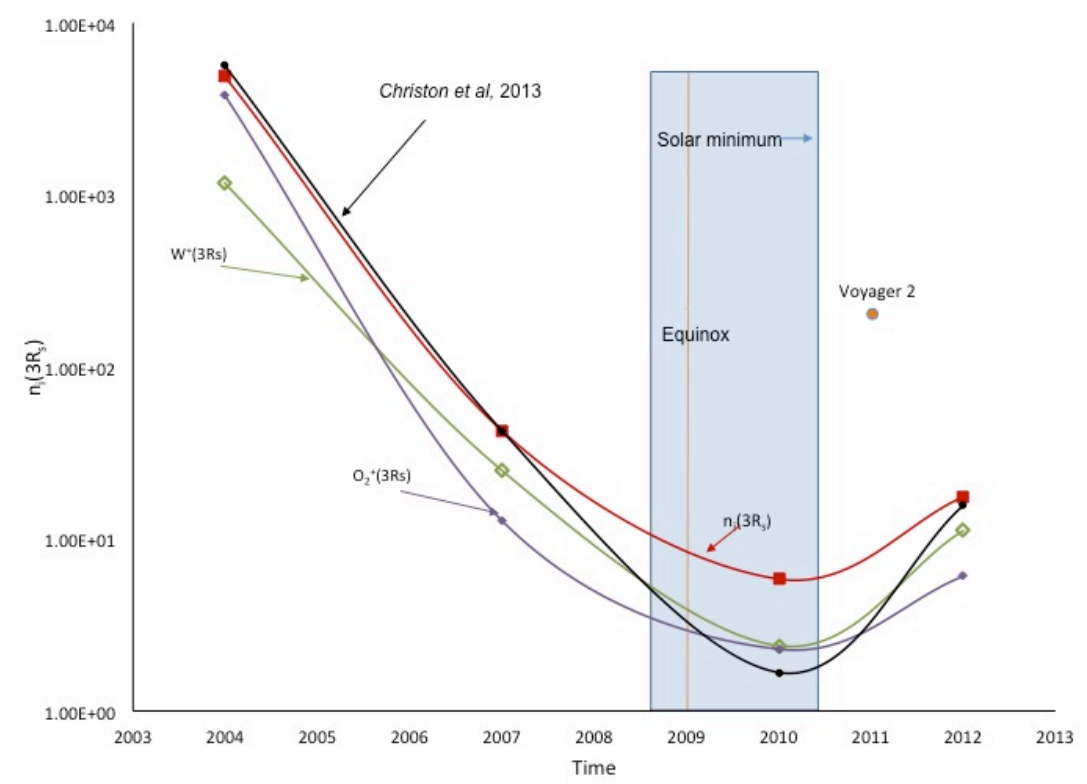

Figure 4. Extrapolated densities at $3 R_{S}, n_{i}\left(3 R_{S}\right)$ vs. year, obtained from the value of $c$ from the fits in Figs. 2 and 3: (red) total heavy ion density from Fig. 2; (purple) $\mathrm{O}_{2}{ }^{+}$and (green) $\mathrm{W}^{+}$from Fig. 3. Lines are drawn to guide the eye. Using powers of 9.8 and 14.1, which gave slightly better fits to the $\mathrm{O}_{2}{ }^{+}$and $\mathrm{W}^{+}$respectively changed these values by $\sim 10 \%$. (Black line) $\mathrm{O}_{2}{ }^{+} / \mathrm{W}^{+}$ratio from MIMI data (Christon et al., 2013) times our $\mathrm{W}^{+}$data. Red dot: Voyager 2 total ion density Aug. 1981, one year after equinox but close to solar maximum. Orange vertical line marks equinox in August of 2009. The grey rectangle marks the region of solar minimum: late 2008 through mid-2010.

In Fig. 4 we plot the fit coefficients, $c$, from Figs. $2 \& 3$. These are essentially the

155 extrapolated values of the averaged density for each year in the middle of the radial range

156 considered here, $n_{i}\left(3 R_{S}\right)$. Since the ionization rate throughout much of our region is

157 dominated by photo-ionization (e.g., Sittler et al., 2008) we also indicate the rough extent

158 of solar minimum by the grey band and equinox by the vertical line. It is seen that these

159 densities all drop from closest to solstice in 2004 through 2010, closest to equinox and

160 roughly within the solar minimum, and then increase again in 2012. It is also seen that 
$161 \mathrm{O}_{2}^{+}$dominates at SOI, whereas $\mathrm{W}^{+}$dominates in the later years (Elrod et al., 2012)

162 allowing for the significant scatter in the data. A fraction of the $\mathrm{O}_{2}{ }^{+}$and $\mathrm{W}^{+}$produced

163 throughout the magnetosphere gets accelerated to high energies. Therefore, Christon et

164 al. (2013) also saw a clear seasonal dependence in the $\mathrm{O}_{2}^{+} / \mathrm{W}^{+}$ratio in the MIMI data.

165 Rather than compare their ratio to our densities, we multiply our $\mathrm{W}^{+}$data by the ratio and

166 display it in Fig. 4. It is seen that the trends are very similar and the post-equinox increase

167 appears to be delayed in both sets of data. This delay is at least in part due to the fact that

168 the northern illumination was predicted to produce a less robust extended ring

169 atmosphere than southern illumination at the same solar inclination angle (Tseng et al.,

170 2010).

171 Since the recent period of solar minimum occurred close to equinox, the EUV/UV

172 ionization rate also goes through a minimum in the time period indicated in Fig. 4 . The

173 photo-ionization rate of $\mathrm{H}_{2} \mathrm{O}$ can therefore change by a factor of the order of few (e.g.,

174 Huebner et al., 1992). Since solar maximum occurred in about 2000, clearly some of the

175 variation seen in Fig. 4 is due to the changing ionization rate from near the middle of the

176 solar cycle at SOI to a minimum from late 2008 through mid 2010 and then increasing in

177 2011. Because the EUV/UV photons also determine the photon-induced decomposition

178 of the icy ring particles, the extended the ring atmosphere varies in this time period

179 somewhat faster than predicted in Tseng et al. (2010). However, the drop in the thermal

180 plasma density in Fig. 4 is much too large to be accounted for by the change in the solar

181 flux alone. To help separate the seasonal and solar cycle effects, we include the equatorial

182 Voyager 2 plasma density taken about a year after equinox in August 1981, but close to

183 solar maximum (Elrod et al., 2012). Although the state of the magnetosphere differed, 
184 affecting the electron impact contribution to ionization, and the instrument sensitivities

185 differ, the comparison suggests that the solar ionizing flux is important, but is not the

186 dominant cause of the temporal variations in the thermal plasma seen in Fig. 4.

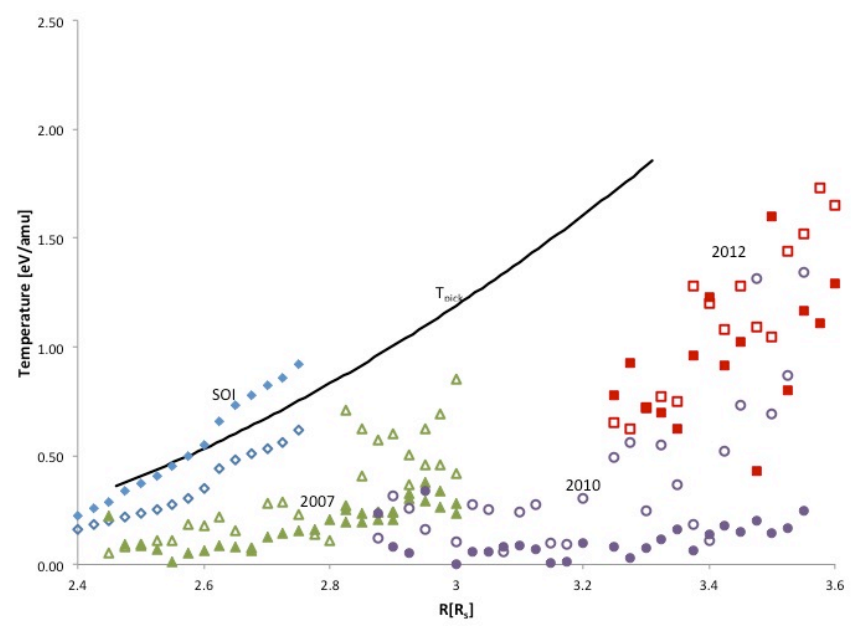

188 Figure 5. Extracted ion temperatures in $(\mathrm{eV} / \mathrm{amu})$ vs. $\mathrm{R}$ in $R_{S}$ : for $\mathrm{O}_{2}^{+}$and $\mathrm{W}^{+}$as indicated. Solid line: pick189 up temperature/amu for ions freshly formed from a neutral in a circular orbit. The significant scatter in the 190 data is roughly consistent with the uncertainties, not shown for clarity.

191

In Fig. 5 we show the ion temperatures, $T_{i}$, for the two species determined from

193 the peak widths. Because the $\mathrm{O}_{2}{ }^{+}$and $\mathrm{W}^{+}$peaks in the CAPS singles data have

194 considerable overlap (Elrod et al., 2012; Elrod 2013) there is a significant amount of

195 scatter in these results. However, the trend is clear. At SOI the $\mathrm{O}_{2}{ }^{+}$temperatures are close

196 to the pick-up temperature calculated for ions freshly formed from neutrals in roughly

197 circular, Keplerian orbits. This indicates the lifetimes are short compared to collisional

198 cooling, reaction and recombination processes. This is not the case for the other years for

199 which the temperatures for each species are a fraction of the pick-up temperature, a trend

200 that continued in our most recent 2012 data. As proposed in Tseng et al. (2013a), this is

201 due to the rapid ion-neutral interactions in this region where the relative ion-neutral

202 speeds are relatively low. 


\section{3. Discussion of the Results}

205 Earlier we developed a one-box homogeneous ion chemistry model to account for

206 the complex and highly variable plasma environment (i.e. density, composition, and

207 temperature) observed near equator between the main rings and Enceladus (Tseng et al.,

208 2013a). We used models of the Enceladus torus as a source for the water group ions, the

209 scattered ring atmosphere and the Enceladus torus as sources of the light ions, $\mathrm{H}^{+}$and

$210 \mathrm{H}_{2}{ }^{+}$, and the scattered ring atmosphere as a source for $\mathrm{O}^{+}$and $\mathrm{O}_{2}{ }^{+}$. The density of the

211 Enceladus torus, although varying in its orbit, does not appear to have a seasonal

212 variability (Smith et al., 2010), but the extended ring atmosphere was predicted to vary

213 due to the changing orientation of the ring plane to the flux of solar UV photons as Saturn

214 orbits the sun (Tseng et al., 2010). Here we again use this model and refer the reader to

215 Tseng et al. (2013a) for the list of reactions and other details.

216 Solving a set of chemical rate equations, we showed that the observed temporal

217 variations in the near equatorial average in the total heavy ion density and $\mathrm{O}_{2}{ }^{+}$density

218 were primarily seasonal due to the predicted variation in the ring atmosphere. The model

219 also required an enhancement at SOI caused by a compressed magnetosphere producing

220 an enhanced hot electron component. Due to the low relative ion speeds in this region,

221 the ion-neutral collisions determined both the composition and the surprisingly low ion

222 temperatures found after SOI as seen in Fig. 5. Even though $\mathrm{O}_{2}$ from the extended ring

223 atmosphere is the dominant source of $\mathrm{O}_{2}{ }^{+}$at SOI, we also showed that $\mathrm{O}_{2}{ }^{+}$is formed by

224 ion-molecule reactions in the magnetosphere, so is present at some level even where the

225 contribution from the extended ring atmosphere is small. Using the dust densities in this 
226 region detected by the Cassini Cosmic Dust Analyzer (CDA)(Kempf et al., 2008), the

227 effect of the ion-dust interactions was found to be unimportant.

228 Our chemical model was applied at an average radial distance, $\sim 3 R_{S}$, and, as

229 stated above, appears to account for the averaged plasma densities, composition and

230 temperatures as a function of the season. However, the radial dependence of the ion

231 densities was not examined. Here we show that in going from southern solstice through

232 equinox and onto illumination of the northern side of the ring plane (2004-2012) the

233 radial dependence of these yearly averaged total ion density increases with increasing

234 distance from Saturn as seen in Figs. 2 and 3. Surprisingly, this was found to be the case

235 whether the plasma was dominated by $\mathrm{O}_{2}^{+}$, as at $\mathrm{SOI}$, or by $\mathrm{W}^{+}$. This dependence is much

236 steeper than the radial dependence of the neutral density in the Enceladus torus (Cassidy

237 and Johnson, 2010) and is opposite to the radial dependence of the extended ring

238 atmosphere (Tseng et al., 2010). Using even a simple model of reaction pathways, which

239 includes a diffusion time, and the neutral sources, the resulting ion density at equinox

240 varies only slowly with $R$ when using the Enceladus torus source in Cassidy and Johnson

241 (2010). Since the average ion densities were about right, either the radial dependence of

242 neutral sources are significantly in error or a quenching mechanism that becomes more

243 important with decreasing distance from the main rings is absent or incorrect.

244 The difference in the spatial morphology of the source rate and the heavy ion

245 density is especially evident in the SOI data when the plasma is heavily dominated by

$246 \mathrm{O}_{2}^{+}$with temperatures close to the pick-up energy. The latter indicates, as stated above,

247 that these ions, formed from oxygen in the extended ring atmosphere, have short

248 lifetimes. The neutral oxygen source clearly decreases with distance from the main rings. 
249 However, it is seen in Fig. 3a that the $\mathrm{O}_{2}{ }^{+}$density increases with increasing $R$ over the

250 narrow range of $R$ for which there is data. Below we consider those processes that might

251 produce the observed radial dependences from 2004 to 2012.

\section{4. Radial Dependence}

253 We focus here on the radial variation in the near equatorial densities in Fig. 2.

254 Although the heavy ion data in 2010 , nearest to equinox varies between $\sim 4$ to $\sim 40 / \mathrm{cm}^{3}$ in

255 going from $\sim 2.9$ to $\sim 3.5 R_{s}$, the ion-chemistry model in $T$ seng et al. (2013a) gives heavy

256 ion densities that are nearly independent of $R\left(\sim 25 / \mathrm{cm}^{3}\right.$ with a slight minimum at $\left.2.75 R_{s}\right)$.

257 Below we discuss the possible reasons for the difference between the model and

258 measured radial dependence. Before proceeding, we note that heavy ion density in a flux

259 tube (density vertically integrated along a field line) at each $R$ is more closely related to

260 the ionization source rate than is the equatorial density. It can be roughly estimated as the

261 equatorial density times the scale height which is proportional to $\sim T_{i}^{1 / 2}$. Because the ion

262 temperature in Fig. 5 increases with increasing $R$, the flux tube content would exhibit an

263 even steeper radial dependence than the near equatorial densities in Fig. 2. Therefore, the

264 difference in the morphology of the heavy ion flux tube content and the spatial

265 morphology of the sources would be even more dramatic.

266 In Tseng et al. (2013a) the averaged ion-molecule collisions and ion-electron

267 recombination processes had timescales near equinox of $\mathrm{a} \sim 10^{5} \mathrm{~s}$ throughout this region

268 and $\sim 10^{4} \mathrm{~s}$ at SOI inside of $\sim 2.8 R_{S}$. Therefore, processes that might account for the steep

269 decay with decreasing $R$ must have shorter time scales. For example, using the fit to the

2702010 data in Fig. 2, a quenching process that accounts for the observed radial dependence

271 can have a longer time scale, greater than a few times $10^{5} \mathrm{~s}$ at $\sim 3.5 R_{S}$. However, at $\sim 2.5 R_{S}$ 
272 our data would require a time scale $\sim 5 \times 10^{4} \mathrm{~s}$. That is, to account for the steep radial

273 dependence, the lifetime of the heavy ions produced must be smallest closest to the edge

274 of the rings. Although we do not determine the exact quenching mechanism, we examine

275 a number of possible processes: enhanced radial diffusion with ions quenching rapidly on

276 the edge of the main rings; the presence of an increased density of small charged grains

277 on which the ions neutralize; enhanced $\mathrm{H}$ or $\mathrm{H}_{2}$ emanating from Saturn's atmosphere or

278 the ring atmosphere increasing the light ion density and quenching the heavy ions; an

279 increased electron-ion recombination rate closer to the rings due to larger electron density

280 and/or smaller electron temperatures.

\section{Quenching by Diffusion}

282 The rate of plasma diffusion in the region of interest has not been examined in

283 any detail. In Tseng et al. (2013a) we used a diffusion model consistent with data taken

284 primarily outside the orbit of Enceladus (e.g., Rymer et al., 2008; Sittler et al., 2008).

285 Extrapolating this model into our region, we used an average diffusion time of $\sim 5 \times 10^{6}$ s.

286 Such times are much too long to affect the radial dependence in the ion density extracted

287 here. Further, due to the predicted radial dependence near the magnetic equator $\left(\sim R^{-3}\right)$,

288 larger times occur at the smallest $R$, opposite to what would be required to explain our

289 results. Finally, we also note that the ion temperatures vs. $R$ in Fig. 5, particularly for

2902007,2010 , and 2012 data, are inconsistent with rapid inward diffusion.

291 Of course, the standard diffusion model might not be applicable in the region of

292 interest. That is, there are significant and day/night asymmetries observed in the inner

293 magnetosphere that are suggestive of an unexplained noon to midnight electric field

294 (Homlberg et al., 2013) or may related to the orbital varying Enceladus source. Although 
295 the observations in the region of interest are sparse, variations in the ion speeds are

296 comparable to the co-rotation speed and could enhance the effective diffusion rate. For

297 instance, Farrell et al. (2008) interpreted their 'down-drifting z-mode tones' over the A-

298 ring, measured by the Cassini RPWS instrument at SOI, as being due to rapid unloading

299 of the plasma onto particles in the A-ring. They presumed the plasma diffused inward

300 from the Enceladus torus source and quenched near the edge of the main rings at a rate of

$301 \sim 40 \mathrm{~kg} / \mathrm{s}$. This would be a significant fraction of the total ion production in the torus

302 inside the orbit of Enceladus. This, in turn, would require diffusion times much more

303 rapid than those discussed above in order to compete with the electron-ion

304 recombination. Since these measurements were only available at SOI when the ring

305 atmosphere was maximum and the magnetosphere was active, their observations are

306 probably not applicable to others years. Rather, they are more likely consistent with the

307 quenching of the relatively dense plasma formed just outside of the main rings from the

308 extended ring atmosphere, which dominates the Enceladus torus source in the region of

309 interest at SOI (Elrod et al., 2012).

310 Quenching on Small Grains

311 Using the dust density observed by the CDA instrument, the ion-dust interaction

312 did not significantly affect the ion loss or cooling in this region (Tseng et al., 2013a).

313 However, there is likely a significant population of submicron-sized dust under the

314 detection limit of this instrument $(>0.9 \mu \mathrm{m})$. As seen in Fig.1 the region inside $3 R_{S}$,

315 contains the G-ring, the F-ring and the edge of the main rings in which collisions of small

316 icy bodies produce debris (Tiscareno et al., 2013; Attree et al., 2013). This debris can

317 deplete the energetic ions and electrons (Paranicas et al., 2008; Cuzzi and Burns, 1988) 
318 as also seen in the CAPS background radiation (Elrod, 2012). Such debris, in the form of

319 small grains, would be a sink for the thermal plasma with the required radial dependence

320 (highest density at $2.4 R_{S}$ and lowest at $3.8 R_{S}$ ) suggestive of a ring source of small grains

321 rather than an Enceladus source. Modeling indicates that the resulting dust/grain

322 population typically follows a steep size distribution varying as $1 / r_{g}{ }^{a}$ with $a \sim 4-5$ where

$323 r_{g}$ is the grain radius (Kempf et al., 2008). The ion-dust cross section can be written as [ $\pi$

$\left.324 r_{g}^{2}\left(1-U_{g} / E\right)\right]$ where $U_{g}$ is the grain potential, which negative in this region (Jurac et al.,

325 1996). Here $E$ is either the thermal energy or the relative flow energy between the ion and

326 the grain, depending on whether the grain has been accelerated to corotation or is in a

327 Keplerian orbit with a speed relative to the corotating plasma of $\sim 1.5 \times 10^{6} \mathrm{~cm} \mathrm{~s}^{-1}$ at $2.5 R_{S}$.

328 Presuming impacting ions neutralize on these grains, using an intermediate size

329 dependence, $\sim 1 / r_{d}^{4.5}$, and the relative speed above, with a density of $1 \mu \mathrm{m}$ grains of $\sim$

$3302 \times 10^{-9} / \mathrm{cm}^{3}$, the density at $2.5 R_{S}$ would require a contribution from grains with radii down

331 to $\sim 10 \mathrm{~nm}$. However, the resulting density of small grains is large enough to significantly

332 deplete the electrons, so that charged grains would dominate the total negative charge in

333 this region. Evidence for this has not been seen, although if the small grain source was

334 highly variable, it might contribute to the significant variability in the electron density in

335 this region reported in Persoon et al. (2013a).

336 Quenching by Hydrogen from the Main Rings or Saturn

337 In addition to the possible presence of small grains from the main and tenuous

338 rings, the region outside of the main rings has significant levels of neutrals that decrease

339 in density with increasing $R$. These are atoms and molecules from the icy ring particles

340 (e.g., $\mathrm{O}, \mathrm{O}_{2}$ and $\mathrm{H}, \mathrm{H}_{2}$; Johnson et al., 2006a; Tseng et al., 2013b) or $\mathrm{H}$ from Saturn 
341 (Shemansky et al., 2009; Melin et al., 2009; Tseng et al., 2013b). The light neutrals can

342 react with the heavy ions changing the ion composition. However, with the exception of

343 the important reaction, $\mathrm{H}+\mathrm{O}^{+} \rightarrow \mathrm{H}^{+}+\mathrm{O}$, these reactions do not result in a change in the

344 total heavy ion density. Therefore, we find that an increased $\mathrm{H}$ population, which is also

345 less readily ionized, does not appear to have a major effect on the ion density and

346 composition (Tseng et al., 2103a).

347 The presence of additional neutrals can also act to cool the electrons, especially if

348 they are molecular. In Tseng et al. (2013a) we calculate electron density and temperature,

$349 T_{e}$, self-consistently with the production of and cooling of the ions. That is, we allow the

350 photoelectrons produced to interact and cool by collisions with the molecules, hot

351 electrons, and ions. Since the molecular ion neutralization rate increases with increasing

352 electron density and with decreasing $T_{e}$, as $\sim T_{e}^{-1 / 2}$, the presence of additional neutrals as

353 both a source of additional plasma and cooling of the electrons would cause the

354 recombination rate to increase with decreasing $R$. If the enhanced cooling is due to

355 molecules from the main rings produced by solar UV decomposition of ice particles, it

356 would also be seasonal. That is, it would not be consistent with the dependence seen in

357 Fig. 2: i.e., rapid quenching with decreasing $R$ in 2007 when the seasonal ring atmosphere

358 is predicted to be small. It would require a separate source from the edge of the rings,

359 such as the inward diffusing high-energy background radiation. 


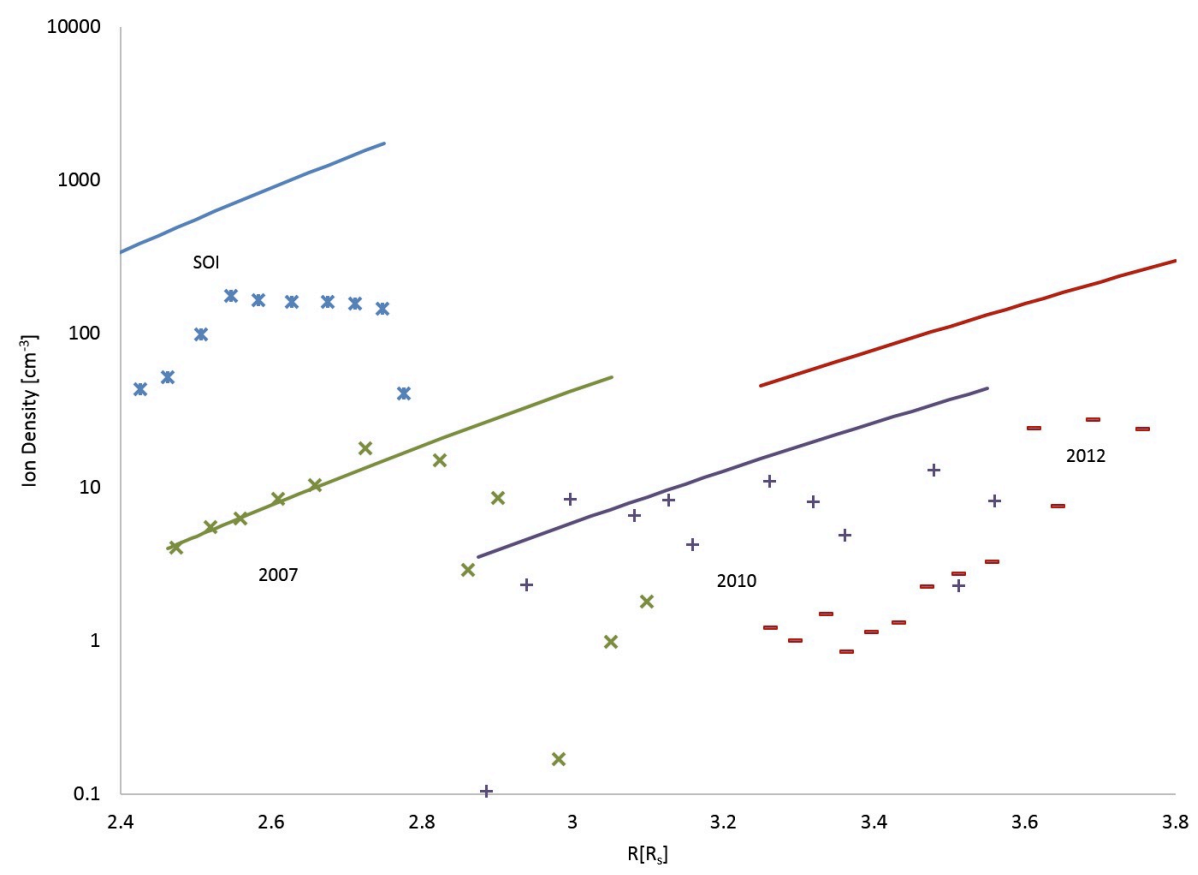

361 Figure 6 . Average ion density vs. $R$. Symbols $\left(\mathrm{x},{ }^{*},+,-\right)$ are the rough estimates of the total light ion $\left(\mathrm{H}^{+}\right.$,

$\left.362 \mathrm{H}_{2}^{+}, \mathrm{H}_{3}^{+}\right)$density, as discussed in the text: colors as in Fig. 2. For comparison, lines are the fits to the heavy 363 ion densities from Fig. 2.

\section{Enhanced Light Ion Density}

As discussed above, the light neutrals can result in the production of light ions,

$367 \mathrm{H}^{+}, \mathrm{H}_{2}{ }^{+}$and $\mathrm{H}_{3}{ }^{+}$. These are not included in the total heavy ion densities so that the radial

368 dependence of the total ion density might differ significantly from that for the heavy ions.

369 In Fig. 6 we use the CAPS data to extract very rough estimates of the light ion densities

370 and overlay them onto the fits for heavy ion densities in Fig. 2. Because the light ion

371 signal usually does not rise above the large background radiation in this region (Elrod

372 2012), these are rough estimates in which a 'feature' in the CAPS data is used when it is

373 seen, but are primarily estimated from the size of the background in the expected location

374 of the peak. Therefore, these results are only for guiding the discussion below.

375 In spite of the significant scatter, it is seen that the light ions apparently can

376 contribute significantly at the smaller values of $R$ in Fig. 5. This is consistent with what 
377 we find using the model in Tseng et al. (2013a). In our chemical model, $\mathrm{H}_{2}$ from the ring 378 atmosphere plays a significant role at SOI, primarily producing, $\mathrm{H}^{+}$and $\mathrm{H}_{2}{ }^{+}$, that account

379 for $>50 \%$ of the total plasma from $2.5-3.0 R_{S}$, larger than our rough estimates at $\mathrm{SOI}$ in

380 Fig. 6. The light ion fraction decreases at larger $R$, roughly consistent with the trend in

381 Fig. 6. Including the light ion contribution produced by $\mathrm{H}_{2}$ from the ring atmosphere, our

382 modeled rates still can not account for the heavy ion radial dependence measured at SOI.

383 Because of the uncertainties in the CAPS light ion densities, accurate

384 measurements of the density and temperatures of the electrons in this region are critical.

385 Persoon et al. $(2013 \mathrm{a}, \mathrm{b})$ recently summarized the RPWS electron data, which they show

386 is highly variable. Averaging over a number of Cassini orbits, their fit to that data inside

387 the orbit of Enceladus suggests a much more slowly varying radial dependence $\left(\sim R^{4}\right.$;

388 Persoon et al. 2013b) than that seen in Fig. 2 in the heavy ion data. However, as shown in

389 Elrod et al. (2012) their densities at SOI are not inconsistent with our ion densities, and

390 there is also rough agreement between their electron densities and the heavy ion densities

391 in Fig. 2 at $\sim 3.5 R_{S}$. This still allows that the electron densities at $\sim 2.5 R_{S}$ could be

392 significantly greater than our measured heavy ion density for passes other than SOI. This

393 would affect the recombination rates and suggest the presence of a significant component

394 of light ions.

395 The 2007 data is interesting in that Cassini came reasonably close to the main

396 rings at a time well past solstice when the contribution of $\mathrm{O}_{2}{ }^{+}$from the ring atmosphere

397 has been predicted to drop significantly (Tseng et al., 2010). It is seen in Fig. 2 that there

398 is significant scatter in the heavy ion data close to the ring, as the densities on the

399 incoming and outgoing passes differ. It is also seen that the light ion densities in 2007 can 
400 contribute significantly to the total ion density at the smallest values of $R$. RPWS electron

401 data in 2007 soon to be published (A. Persoon, personal communication) suggest that

402 inside $\sim 2.9$ Rs, there is an upturn in the electron density near those values of $R$ closest to

403 Saturn where our light ion estimates appear to contribute significantly. As discussed

404 above, such an upturn would be consistent with a source of light ions produced by

405 coming from inside the region examined.

406 5. Summary

407 Including new data from post-equinox orbits, selecting only ion energy spectra

408 which had significant counts above background, and averaging over passes occurring in

409 the same year, we confirm that the near equatorial heavy ion density exhibits a significant

410 temporal variation between 2004 and 2012 with a minimum near 2010. Although the

411 ionization rate and photo-induced decomposition rate change during the solar cycle, the

412 large variation in density and composition from SOI to equinox a seasonal effect appears

413 to dominate. That is, the densities are very high and dominated by $\mathrm{O}_{2}^{+}$nearest to southern

414 solstice, are smallest close to equinox, and begin to increase post equinox, as seen from

415 the fit parameter to the total ion density in Fig. 4. Although there will be no new data

416 from the CAPS instrument to follow the growth to northern solstice, this interpretation

417 appears to be supported by MIMI observations of energetic heavy ions (Christon et al.,

418 2013) and most recently by the electron data (Persoon et al. 2013a). However, with the

419 limited number of orbits in this region that have good data, we allow that other

420 interpretations are possible and the relative importance of the ring atmosphere and solar

421 activity needs to be explored further. 
422 Surprisingly we also show that the heavy ion density exhibits a steep radial

423 dependence over this narrow region (2.4 to $3.8 R_{S}$ ), which appears to be roughly

424 independent of the year. Our ion-neutral chemical model (Tseng et al., 2013a) was able to

425 describe the observed temporal dependence in the average density, composition and

426 temperatures in this region. In this we used a seasonal ring atmosphere source (Tseng et

427 al., 2010) and Enceladus torus source (Cassidy and Johnson, 2010). This model also

428 accounted for the surprisingly low ion temperatures found after SOI, seen again in the

429 recently analyzed 2012 data set (Fig. 5). However, this model could not describe the

430 observed radial dependence of the heavy ion density in Fig. 2, even allowing for the

431 considerable uncertainties.

432 In the absence of the SOI data set, which is dominated by $\mathrm{O}_{2}^{+}$supplied primarily

433 by the ring atmosphere, one might conclude that the average densities in the other years

434 are consistent with an Enceladus neutral torus source that is variable within the range

435 suggested by Smith et al. (2010). In this case SOI would be exceptional, due to a robust

436 ring atmosphere, with the contribution of the extended ring atmosphere negligible in

437 2007-2012. If that is the case, then the radial dependence in the heavy ion density seen in

438 those years might suggest that the models of the Enceladus neutral torus should decay

439 more steeply than predicted with decreasing $R$. Since the distribution of neutrals in the

440 torus depends nonlinearly on the density due to neutral-neutral collisions (Cassidy and

441 Johnson, 2010; Cassidy et al., 2011), if the densities are lower than those used the radial

442 dependence would be steeper, as seen in models that neglect the neutral-neutral collisions

443 (Johnson et al., 2006; Smith et al., 2010). Recently the variability seen by Smith et al.

444 (2010) has been shown to be associated with the position of Enceladus in its orbit 
445 (Hedman et al. 2013), so that the model of the neutral torus used in our ion chemistry

446 model needs updating. Such work is now in progress and includes more detail,

447 accounting for interactions with ice grains, the small moons, and the F \& G rings within

448 this region.

449 It is clear, however, that in spite of the large differences in the magnitude of the

450 ion densities in going from 2004 to 2012, there is a similar radial trend over the narrow

451 region (2.4 to $3.8 R_{S}$ ). We point out that this cannot be due to rapid inward diffusion of

452 ions formed from neutrals in the Enceladus torus, which Farrell et al. (2008) proposed to

453 explain their SOI data. In the absence of good measurements of the light ion and electron

454 densities for all passes, we suggest this dependence is primarily due to enhanced

455 quenching of the heavy ion plasma with decreasing distance from the edge of the A-ring.

456 Unless the ion diffusion rate in this region differs significantly from estimates at larger $R$,

457 the observed dependence is likely due to material emanating from smaller radial

458 distances. Of particular interest is $\mathrm{H}_{2}$, which plays an important role in our chemical

459 model (Tseng et al. 2013a). A likely source is the significant, high-energy, background

460 radiation, which diffuses inward and quenches on the particles in the tenuous rings and at

461 the edge of the A-ring leading to production of $\mathrm{H}_{2}$ from the ice particles.

462 Since there will be no new CAPS data, understanding this neglected, but

463 extremely interesting, region of the Saturnian system will require more detailed modeling

464 of the extended ring atmosphere, the fate of the ring plasma, the role of the tenuous rings,

465 the quenching of the high energy background radiation, and the variability in the

466 Enceladus source in this region. 


\section{6. Acknowledgements}

469 We acknowledge support through Southwest Research Institute from a grant for the

470 Cassini Mission from JPL and by a grant from NASA's Planetary Atmospheres program.

471 This work was also supported in part by National Aeronautics and Space Administration,

472 Langley Research Center, under research cooperative agreement No. NNL09AA00A

473 awarded to the National Institute of Aerospace.

474

$475 \quad 7 . \quad$ References

476 Attree, N.O., C. D. Murray, G. A. Williams, N. J. Cooper, 2013. A Survey of Low-

477 Velocity Collisional Features in Saturn’s F Ring. Astroph-EP. arXiv 1309.3119v1

478 Cassidy, T.A., R.E. Johnson, and A.R. Hendrix, 2011. Collisional Evolution of the

479 Enceladus Neutral Cloud, 27th International Symp. on Rarefied Gas Dynamics, AIP

480 Conf. Proc. 1333, 1133-1138 doi: 10.1063/1.3562796.

481 Cassidy, T.A. and R.E. Johnson, 2010. Collisional spreading of Enceladus' neutral

482 cloud, Icarus 209, 696-703.

483 Christon, S.P., D. C. Hamilton, R. D. DiFabio, D. G. Mitchel, S. M. Krimigis, D. S.

484 Jontof-Hutter, Saturn suprathermal $\mathrm{O}_{2}{ }^{+}$and mass $-28^{+}$molecular ions: Long-term seasonal

485 and solar variation, 2013. J.Geophys. Res. 28 JUN 2013 DOI: 10.1002/jgra.50383.

486 Cuzzi, J.N., and J. A. Burns 1988. Charged particle depletion surrounding Saturn's

487 F ring: Evidence for a moonlet belt? Icarus 74, 284-324

488 Elrod, M.K., W.-L. Tseng, R.J. Wilson, R.E. Johnson, Seasonal Variations in Saturn's

489 Plasma between the Main Rings and Enceladus, 2012. J.Geophys.Res. 117, A03207,

490 doi:10.1029/2011JA017332. 
491 Elrod, M.K. 2012. Seasonal Variations in Saturn's Plasma between the Main Rings

492 and Enceladus PhD Thesis. The University of Virginia, Charlottesville, VA 22904

493 Farrell, W. M., M. L. Kaiser, D. A. Gurnett, W. S. Kurth, A. M. Persoon, J. E. Wahlund,

494 and P. Canu, 2008. Mass unloading along the inner edge of the Enceladus plasma torus,

495 Geophys. Res. Lett., 35, L02203, doi:10.1029/2007GL032306

496 Holmberg, M.K.G., J.-E. Wahlund and M.W. Morooka, 2013. Day/night side asymmetry

497 of ion densities and velocities in Saturn's inner magnetosphere EPSC Abstracts Vol. 8,

498 EPSC2013-519, 2013 European Planetary Science Congress 2013.

499 Hedman, M.M., C. M. Gosmeyer, P. D. Nicholson, C. Sotin, R. H. Brown, R. N. Clark,

500 K. H. Baines, B. J. Buratti, M. R. Showalter, 2013. An observed correlation between

501 plume activity and tidal stresses on Enceladus. Nature 500, 182-184.

502 Johnson, R.E., J.G. Luhmann, R.L. Tokar, M. Bouhram, J.J. Berthelier, E.C. Siler, J.F.

503 Cooper, T.W. Hill, H.T. Smith, M. Michael, M. Liu, F.J. Crary, D.T. Young, Production,

504 Ionization and Redistribution of O2 Saturn's Ring Atmosphere, 2013a. Icarus 180, 393-

505402.

506 Johnson, R.E., Smith, H.T., Tucker, O.J., Liu, M., Burger, M.H., Sittler, E.C., Tokar,

507 R.L., 2006b. The Enceladus and OH Tori at Saturn. Astrophys. J. 644, L137-L139.

508 Jurac, S., R.E. Johnson, R.A. Baragiola and E.C. Sittler, 1995. Charging of ice grains by

509 low-energy plasma: Application to Saturn's E ring, J. Geophys. Res. 100,

$510 \quad 14,821-14,831$.

511 Kempf, S., U. Beckmann, R. Srama, M. Horanyi, S. Auer, E. Grün, 2006 The

512 electrostatic potential of E ring particles. Planet. Space Sci. 54, 999-1006. 
513 Kempf, S.; Beckmann, U.; Moragas-Klostermeyer, G.; Postberg, F.; Srama, R.;

514 Economou, T.; Schmidt, J.; Spahn, F.; Grün, E. 2008. The E ring in the vicinity of

515 Enceladus. I. Spatial distribution and properties of the ring particles. Icarus 193, 420-437.

516 Martens, H.R., D.B. Reisenfeld, J.D. Williams, R.E. Johnson, and H.T. Smith, 2008.

517 Observations of molecular oxygen ions in Saturn's inner magnetosphere,

518 Geophys.Res.Letts. 35, L20103, doi:10.1029/2008GL035433.

519 Melin et al., 2009. The distribution of atomic hydrogen and oxygen in the magnetosphere

520 of Saturn, Planetary and Space Science, 57, 1743-1753.

521 Paranicas, C., D.G. Mitchell, S.M. Krimigis, D.C. Hamilton, E. Roussos, N. Krupp, G.H.

522 Jones, R.E. Johnson, J.F. Cooper, T.P. Armstrong, 2008. Sources and losses of energetic

523 protons in Saturn's magnetosphere, Icarus 197, 519-525.

524 Persoon, A.M., D. A. Gurnett, J.-E. Wahlund, M. W. Morooka, W. S. Kurth, G. B.

525 Hospodarsky, and J. B. Groene. 2013a. Local Time Asymmetry and Seasonal Variations

526 of the Electron Density Distribution in Saturn's Inner Magnetosphere. Poster 2013 AGU

527 Meeting, San Francisco.

528 Persoon, A.M., D.A. Gurnett, J.S. Leisner, W.S. Kurth, J.B. Groene, and J.B. Faden,

529 2013b. The plasma density distribution in the inner region of Saturn's magnetosphere. J.

530 Geophys. Res. In press

531 Rymer et al Rymer, A. M.; Mauk, B. H.; Hill, T. W.; Paranicas, C.; Mitchell, D. G.;

532 Coates, A. J.; Young, D. T., 2008. Electron circulation in Saturn's magnetosphere. J.

533 Geophys. Res. 113, CiteID A01201. doi: 10.1029/2007JA012589

534 Shemansky, D. E.; X, Liu and H. Melin, 2009. The Saturn hydrogen plume, Planet. Space 535 Sci., 57, 1659-1670. 
536 Sittler, E. C., et al., 2008. Ion and neutral sources and sinks within Saturn's

537 inner magnetosphere: Cassini results, Planet. Space Sci., 56, 3-18.

538 Smith, H.T., R.E. Johnson, M.E. Perry, D.G. Mitchell, R.L. McNutt, D.T. Young, 2010.

539 Enceladus plume variability and the neutral gas densities in Saturn's

540 magnetosphere, J.Geophys.Res. 115, A10252, doi:10.1029/2009JA015184.

541 Tiscareno, M. S.; Mitchell, C, J.; Murray, C. D.; Di Nino, D.; Hedman, M. M.;

542 Schmidt, J.; Burns, J. A.; Cuzzi, J. N.; Porco, C. C.; Beurle, K.; Evans, M.W, 2013.

543 Observations of Ejecta Clouds Produced by Impacts onto Saturn's Rings. Science 340,

$544 \quad 460-464$

545 Tokar, R.L., R.E. Johnson et al., 2005. Cassini Observations of the ThermalPlasma in the

546 Vicinity of Saturn's Main Rings and the F and G rings. Geophys. Res. Letts. 32, L14S04,

547 doi:10.1029/2005GL022690.

548 Tseng, W.-L., R.E. Johnson, and M.K. Elrod, 2013a. Modeling the seasonal variability of

549 the plasma environment in Saturn's magnetosphere between main rings and Mimas, Plan

550 \& Space Sci. 77, 126-135.

551 Tseng, W.-L., R.E. Johnson and W.-H. Ip, 2013b.The Atomic Hydrogen Cloud in the

552 Saturnian System, Planet. Space Sci. in press.

553 Tseng, W.-L., . E. Johnson, M. F. Thomsen, T. A. Cassidy and M. K. Elrod, 2011.

554 Neutral $\mathrm{H}_{2}$ and $\mathrm{H}_{2}{ }^{+}$ions in the Saturnian magnetosphere, JGR 116, A03209,

555 doi:10.1029/2010JA016145.

556 Tseng, W-.L., W.-H. Ip, R.E. Johnson, T.A. Cassidy, and M.K. Elrod, 2010. The

557 Structure and time variability of the ring atmosphere and ionosphere, Icarus 206, 382-

558389. 